\title{
Direct transplantation of mesenchymal stem cells into the knee joints of Hartley strain guinea pigs with spontaneous osteoarthritis
}

\author{
Mitsuhiko Sato ${ }^{1}$, Kenzo Uchida1*, Hideaki Nakajima', Tsuyoshi Miyazaki ${ }^{1}$, Alexander Rodriguez Guerrero', \\ Shuji Watanabe ${ }^{1}$, Sally Roberts ${ }^{2}$ and Hisatoshi Baba
}

\begin{abstract}
Introduction: Mesenchymal stem cells (MSCs) can differentiate into various connective tissue cells. Several techniques have been used for the clinical application of MSCs in articular cartilage repair; however, there are many issues associated with the selection of the scaffold material, including its ability to support cell viability and differentiation and its retention and degradation in situ. The application of MSCs via a scaffold also requires a technically demanding surgical procedure. The aim of this study was to test the outcome of intra-articular transplantation of mesenchymal stem cells suspended in hyaluronic acid (HA) in the knee joints of Hartley strain guinea pigs with spontaneous osteoarthritis (OA).

Methods: Commercially available human MSCs were cultured, labeled with carboxyfluorescein diacetate succinimidyl ester (CFDA-SE), suspended in either PBS or HA, and injected into the knee joints of 7-month-old animals. The control animals were injected with either PBS or HA alone. The animals were sacrificed at 1, 3, and 5 weeks post transplantation, the knee joints harvested, and fluorescent microscopic analysis was performed. Histological and immunohistochemical analysis were performed at 5 weeks post transplantation.

Results: At 5 weeks post transplantation, partial cartilage repair was noted in the HA-MSC group but not in the other groups. Examination of CFDA-SE-labeled cells demonstrated migration, differentiation, and proliferation of MSC in the HA-MSC group. There was strong immunostaining for type II collagen around both residual chondrocytes and transplanted MSCs in the OA cartilage.

Conclusion: This scaffold-free and technically undemanding technique appears to result in the regeneration of articular cartilage in the spontaneous OA animal model. Although further examination of the long-term effects of transplantation is necessary, the findings suggest that intra-articular injection of HA-MSC mixture is potentially beneficial for OA.
\end{abstract}

\section{Introduction}

Osteoarthritis (OA) of the knee joint is characterized pathologically by degeneration of articular cartilage, sclerosis of the subchondral bone, and marginal osteophyte formation, and is characterized clinically by chronic devastating pain and disability in the elderly. $\mathrm{OA}$ is a major public health problem and its prevalence is expected to increase dramatically and rapidly over the

\footnotetext{
* Correspondence: kuchida@u-fukui.ac.jp

'Department of Orthopaedics and Rehabilitation Medicine, Faculty of Medical Sciences, University of Fukui, Matsuoka-Shimoaizuki 23, Eiheiji, Fukui 910-1193, Japan

Full list of author information is available at the end of the article
}

next 20 years with an increasingly aged population [1]. Although tibial osteotomy and total knee arthroplasty have been pursued in a large number of patients to eliminate joint pain and improve joint function, the majority of patients with knee OA are managed conservatively with medication and/or physiotherapy. Development of less technically demanding but effective therapies for knee OA, such as cell transplantation with or without scaffold enhancement, is therefore desirable.

Mesenchymal stem cells (MSCs) have the capacity to differentiate into a variety of connective tissue cells [2-6]. Several techniques have been used for the clinical 
application of MSCs in articular cartilage repair [7,8]. In general, the cells are delivered into either the cartilage or bone using a three-dimensional scaffold fixed to the articular defect site. There are many issues associated with the selection of the scaffold material, however, including its ability to support cell viability and differentiation and its retention and degradation in situ. Moreover, the application of MSCs via a scaffold usually requires a technically demanding surgical procedure. On the other hand, direct intra-articular injection of MSCs has only been carried out in a limited experimental setting $[9,10]$. In these animal studies, autologous MSCs mixed with a dilute solution of sodium hyaluronan (hyaluronic acid (HA)) as a cell binding or cytotactic factor were directly injected into the knee joint of surgically induced knee OA or focal cartilage defect in certain animal models. The procedure resulted in retardation of the progression of destruction of the degenerative cartilage. Although the injection of MSCs in HA may be the simplest approach clinically, disease progression is rapid in these models, thus making it less amenable to therapeutic intervention [11]. The potential outcomes of this method as a treatment for the slowly progressive process of cartilage degeneration, as commonly occurs in human OA, are still unknown.

The objective of the present study was to determine whether intra-articular injection of MSCs suspended in HA solution into the knee joint enhances the repair of degenerated cartilage in an animal model of spontaneous OA. We used Hartley strain guinea pigs because these animals spontaneously develop degenerative cartilage changes in the knee joint that mimic those of human OA [11-13]. The disease is generally bilaterally symmetrical on the medial tibial plateau in an area unprotected by the meniscus, and the earliest changes can be seen when animals are approximately 3 months old $[12,13]$. This animal model is more suitable for the evaluation of disease-modifying treatments.

\section{Materials and methods}

\section{Experimental animals}

Seven-month-old male Hartley strain guinea pigs $(n=$ 60) weighing $928 \pm 4.8 \mathrm{~g}$ (mean \pm standard deviation) were used. The experimental protocol met the Animal Care Ethics Committee Guidelines for Experimental Studies of Fukui Medical University and Institutional Review Board Guidelines for Stem Cell Research.

\section{Preparation of human MSCs for in vivo tracing}

Human MSCs were purchased from Lonza (lot number PT-2501; Lonza, Walkersville, MD, USA). The cells were cultured at $37^{\circ} \mathrm{C}$ in a humidified atmosphere of $5 \%$ $\mathrm{CO}_{2}$ in mesenchymal stem cell growth medium (MSCGM $^{\mathrm{TM}}$; Lonza). The medium was replaced every 3 days. Seven to 10 days after incubation, the cells reached $75 \%$ confluence in the seeded flask and were subcultured. Cells from passages three to five were used for the experiments. For in vivo tracing, the MSCs were labeled with carboxyfluorescein diacetate succinimidyl ester (CFDA-SE) (Vybrant CFDA SE Cell Tracer Kit (V12883); Molecular Probes, Eugene, OR, USA) as described previously [14].

\section{Intra-articular injection of MSCs}

The labeled cells were carefully introduced into a new syringe together with $2 \mathrm{ml} \mathrm{HA}$ (molecular weight $8 \times$ $10^{5}$, Artz $^{\circledR}$; Seikagaku Kogyo, Tokyo, Japan), and the cell suspension containing $7.0 \times 10^{6}$ labeled MSCs was injected into the medial compartment of the left knee joint of 7-month-old guinea pigs. For this purpose, an 18 -gauge needle was inserted posterior to the medial edge of the patellar ligament, through the triangle formed by the epicondyle of the femur, the meniscal/ tibial plateau, and the notch formed by their junction.

\section{Subtyping of animal groups and histological samples}

The animals were divided into four treatment groups at random: PBS group $(n=15)$, HA-injected group $(n=$ $15)$, PBS + MSC group $(n=15)$, and HA + MSC group $(n=15)$. The control groups were similar to the test groups with respect to age and weight. In each group, the animals were sacrificed at 1,3 , and 5 weeks post transplantation of cells and/or carrier. The proximal tibia was dissected out and prepared for examination.

\section{Macroscopic examination}

The surface of the distal head of the femur and the tibial plateau were exposed. India ink $(2 \mathrm{ml})$ was injected onto the joint surface using a syringe, and 1 minute later the surfaces were washed with physiological saline. The staining pattern of the cartilage surface was observed macroscopically. The gross findings were classified and scored into six grades according to the assessment suggested by Hayashi and colleagues [15]. The assessment was conducted by two independent examiners (HN, TM), who were blinded to each other's findings and to the treatment group assignment of the animals. Finally, the scores evaluated by these two examiners were averaged to obtain the overall score.

\section{Histological and immunohistochemical examinations}

The proximal tibia was harvested and fixed in $10 \%$ buffered formaldehyde solution at $4^{\circ} \mathrm{C}$ for 48 hours. The samples were snap frozen in liquid nitrogen and then embedded in optimal cutting temperature compound. The frozen blocks were cut into sections $10 \mu \mathrm{m}$ thick according to the methods described previously [16]. Serial sections $10 \mu \mathrm{m}$ thick, 20 frontal sections in each 
knee, were prepared carefully in order to include the severely degenerated area not covered by meniscus. Sections from each animal were used for histological, immunohistochemical, and fluoroscopic analyses.

The sections were stained with $\mathrm{H} \& \mathrm{E}$ and safranin $\mathrm{O}$ using standard methods. We assessed the severity of knee OA using the modified Mankin criteria described by Armstrong and colleagues [17], expressed as the OA score. All sections were graded by three independent observers blinded to the treatment group, and the median score was used for statistical analysis.

The tissue sections were also stained with a primary polyclonal antibody, raised in rabbits against type II collagen (dilution 1:200; COSMO BIO, Tokyo, Japan), for 1 hour at room temperature. After rinsing in PBS, the tissue was incubated with biotinylated horse anti-rabbit IgG secondary antibody for 30 minutes at room temperature. Immunohistochemical staining was detected with VECTA STAIN ABC Reagent (Vectastain Elite Kit; Vector Laboratories, Burlingame, CA, USA), followed by staining with 3,3-diaminobenzidine tetrahydrochloride (Dojin Chemicals, Tokyo, Japan). In addition, to evaluate the location of collagen type II relative to the cells, other sections were incubated with the secondary antibody for goat anti-rat Alexa Flour ${ }^{\circledR} 568$-conjugated antibody (1:250; Molecular Probes) for 1 hour at room temperature. These sections were counterstained with the nuclear marker 4',6-diamidino-2-phenylindole.

Cells labeled with CFDA-SE can be visualized by fluorescence microscopy using standard fluorescein filter sets. The approximate excitation and emission peaks of this product after hydrolysis are $492 \mathrm{~nm}$ and $517 \mathrm{~nm}$. The labeled cells were quantified in $500 \times 500 \mu \mathrm{m}^{2}$ areas of tibial frontal section (original magnification, $\times 100)$.

\section{Immunoblot analysis}

Immunoblot analysis was carried out with 15\% SDSPAGE. Total protein $(80 \mu \mathrm{g} / \mathrm{lane})$ extracted from the cartilage was transferred onto polyvinylidene difluoride membrane for 70 minutes using a semi-dry blot apparatus. The membrane was washed twice in PBS containing $0.05 \%$ Tween 20 , blocked by $5 \%$ skimmed milk in PBS for 1 hour at room temperature, and then incubated with the collagen type I, collagen type II, or matrix metalloproteinase-13 primary antibody (dilution 1:20; COSMO BIO) overnight at $4^{\circ} \mathrm{C}$, followed sequentially by anti-mouse IgG antibody and avidin-biotinylated peroxidase complex (for 30 minutes each). After triple washing in $0.1 \mathrm{M} \mathrm{PBS}$, the membrane was incubated in enhanced chemiluminescence reagent for 1 minute, and then the antibody binding was visualized using a FluorChem ${ }^{\mathrm{TM}}$ 8000 System (Alpha Innotech Corporation, San Leandro, CA, USA). The size of each band was normalized to $\beta$ - actin (lot number IMG-5142A, 1:1,000; Imgenex, San Diego, CA, USA).

\section{Statistical analysis}

Data are expressed as the mean \pm standard deviation. Differences between groups were examined for statistical significance using one-way analysis of variance followed by the Bonferoni/Dunn post-hoc paired test for comparison between groups. $P<0.05$ denoted the presence of a significant difference between groups. The above tests were conducted using the Statistical Package for Social Sciences (version 11.0; SPSS Inc., Chicago, IL, USA).

\section{Results}

\section{Assessment of xenogeneic transplantation of MSCs}

Clinically, all guinea pigs tolerated the cell injection, and there was no evidence of local inflammation, joint effusion, or unloading of the joint resulting from the cell treatment. Histologically, the frontal section of the entire knee joint showed no evidence of synovial hyperplasia (Figure 1A, B, C). Fluorescence examination showed labeled MSCs in the fibrillated cartilage at the medial tibial plateau (Figure 1D'). A few labeled cells were also detected in the synovial lining and medial meniscus (Figure 1B', C'), but no such cells were found in the lateral region of the articular cartilage and meniscus (Figure 1E').

\section{Macroscopic findings}

No India ink staining was observed in the lateral tibial plateau of any specimen. In the PBS, HA, and PBS + MSC groups, the surfaces of the articular cartilage over the bare medial tibial plateau (not covered with the meniscus) were rough and strongly stained at 5 weeks post transplantation (Figure $2 \mathrm{~A}, \mathrm{~B}, \mathrm{C}$ ). In the $\mathrm{HA}+$ MSC group, the surface of the medial plateau was relatively smooth and the staining intensity was weaker in the same time interval (Figure 2D). The macroscopic OA score was significantly lower in the HA + MSC group than in the other groups (Figure 2E).

\section{Histological findings}

The PBS and PBS + MSC groups showed depletion of chondrocytes (which extended to the transitional zone) and matrix fibrillation (which extended from the transitional zone to the radial zone) (Figure 3A, C). The safranin-O-stained matrix was reduced in all treatment groups with the exception of the HA + MSC group (Figure $3 \mathrm{E}, \mathrm{G})$. In the HA group, matrix fibrillation of the articular surface did not extend to the radial zone (Figure $3 \mathrm{~B}$ ) and weak matrix staining and cell depletion were observed (Figure 3F). The HA + MSC group showed large numbers of chondrocytes with cluster formations in the radial layer of the articular cartilage. 

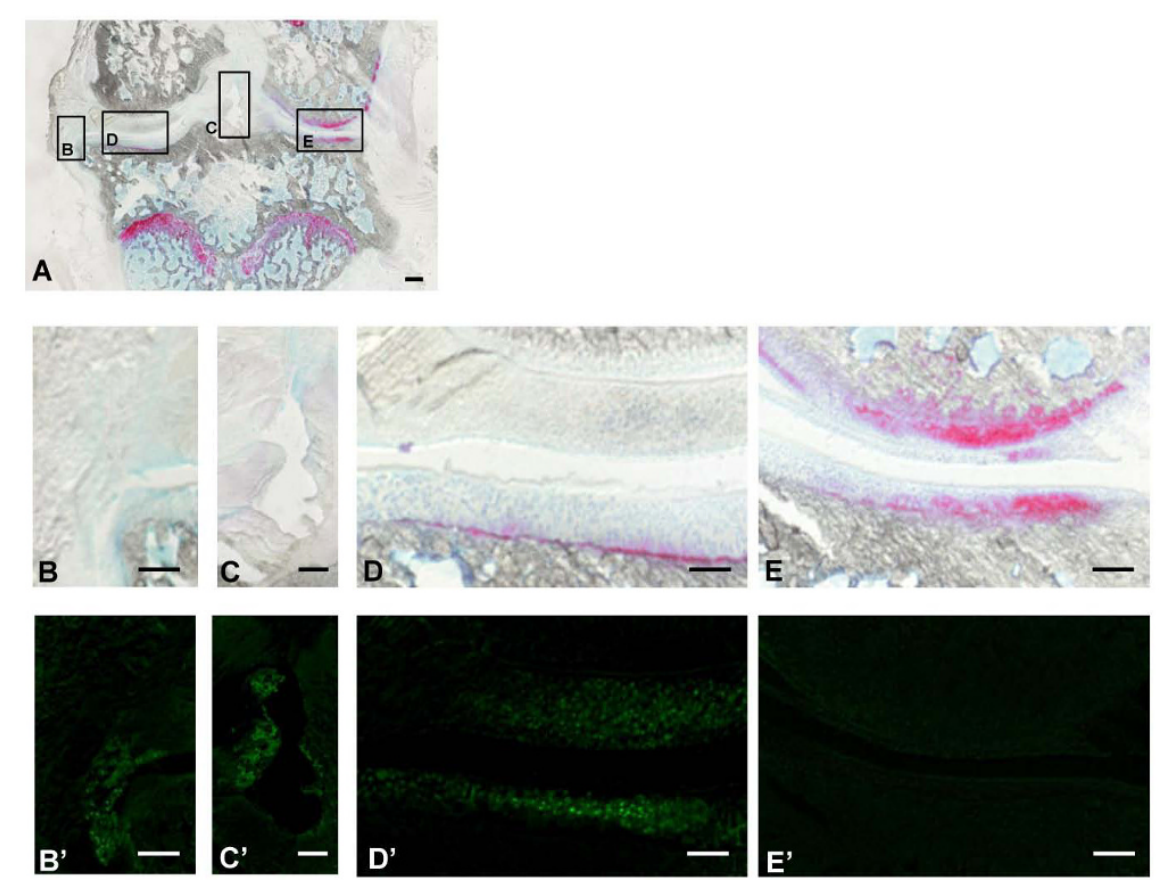

Figure 1 Xenogeneic transplantation of mesenchymal stem cells. (A to E) Photomicrographs showing frontal sections, $10 \mu \mathrm{m}$ thick, of the whole knee joint stained with safranin O. (B' to $E^{\prime}$ ) Fluorescent microscopy for in vivo tracing of injected mesenchymal stem cells (MSCs). Bar = $500 \mu \mathrm{m}(\mathrm{A}), 200 \mu \mathrm{m}$ (B to $E_{1} B^{\prime}$ to $\left.E^{\prime}\right)$.

Furthermore, the matrix around the cell clusters was
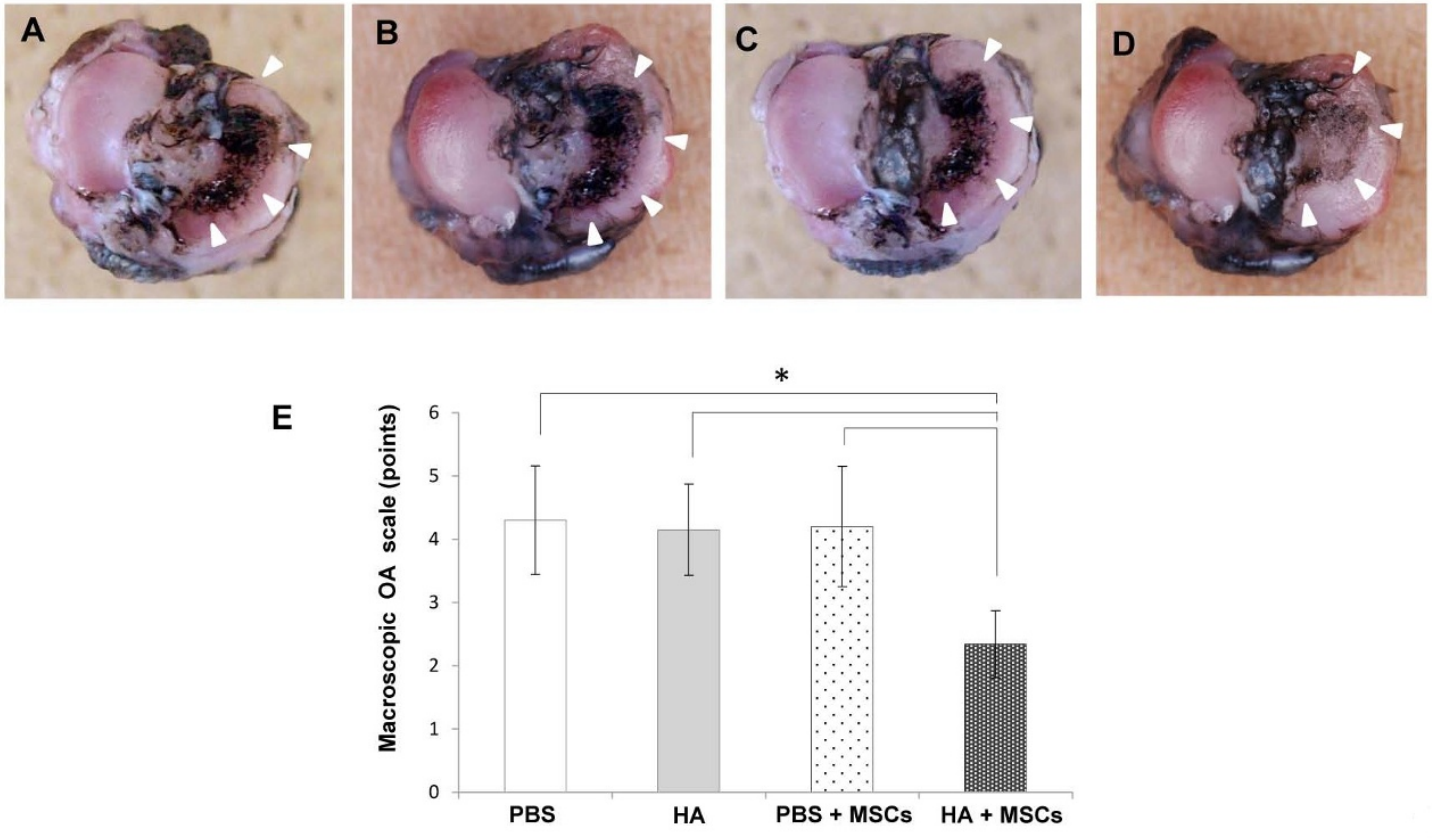

Figure 2 Macroscopic findings. Macroscopic photographs (bird's-eye view) of the articular surface of the tibial plateau 5 weeks after injection of each reagent in Hartley strain guinea pigs, stained with India ink: (A) PBS group, (B) hyaluronic acid (HA) group, (C) PBS + mesenchymal stem cell (MSC) group, (D) HA + MSC group. Arrowheads: stained lesions. (E) Macroscopic osteoarthritis (OA) scores in the same animal groups evaluated 5 weeks after injection. Data are mean \pm standard deviation of five guinea pigs in each group. ${ }^{*} P<0.05$. 


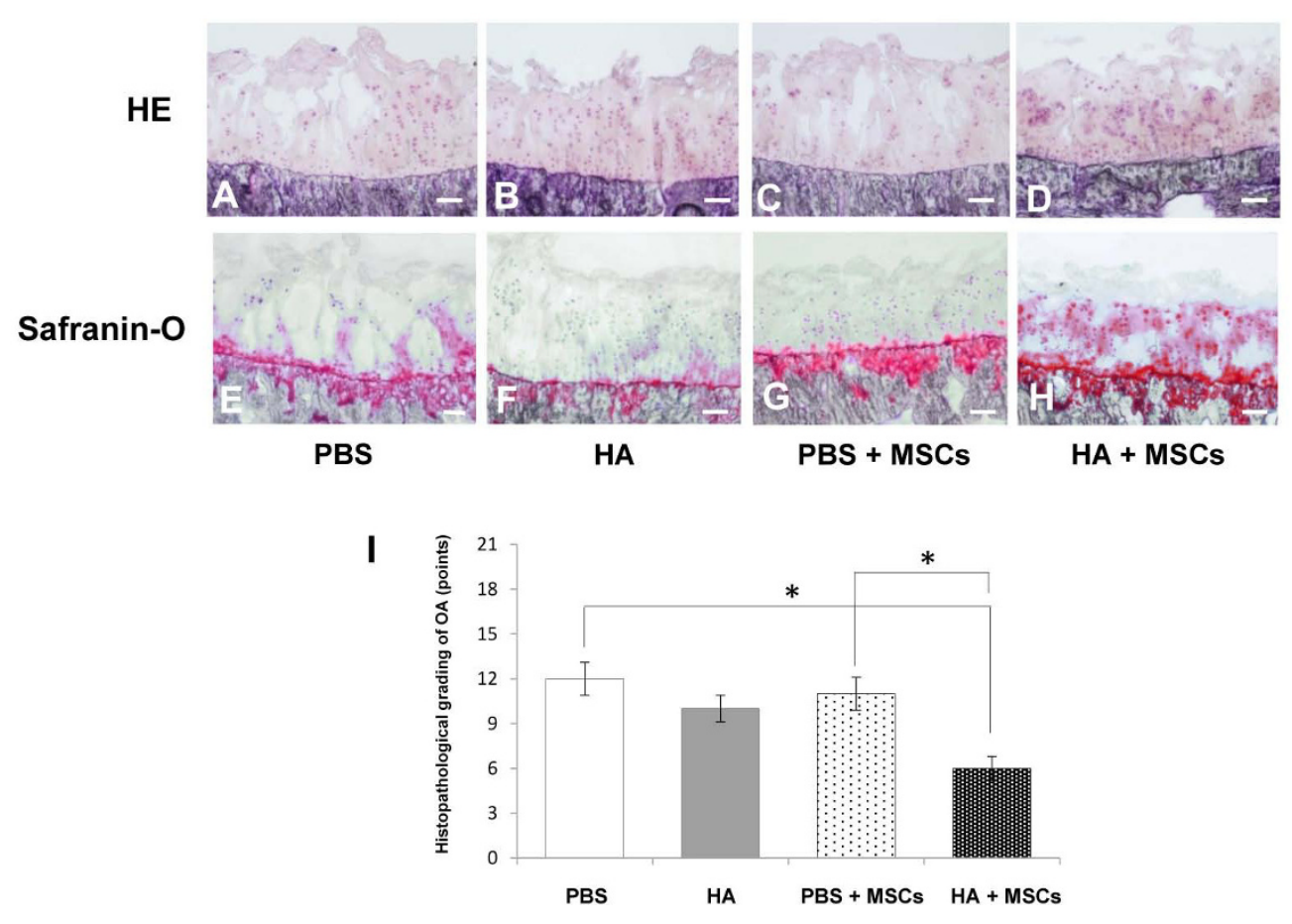

Figure 3 Histological findings. Photomicrographs showing serial frontal sections $10 \mu m$ thick stained with (A to $\mathbf{D}) \mathrm{H} \& \mathrm{E}$ and (E to $\mathbf{H}$ ) safranin O. Bar $=100 \mu \mathrm{m}$ in all sections. (I) Histological grade of knee osteoarthritis assessed by modified Mankin criteria 5 weeks after injection, according to the treatment modality. Data are mean \pm standard deviation of five guinea pigs in each group. ${ }^{*} P<0.05$. HA, hyaluronic acid; MSC, mesenchymal stem cell.

strongly stained in safranin-O-stained sections at 5 weeks post transplantation (Figure 3D, H).

At 5 weeks post transplantation, significant differences were noted between the OA scores of the PBS and HA + MSC groups, and between the scores of PBS + MSC and HA + MSC groups (Figure 3I).

\section{Immunohistochemical and immunoblot analyses}

The immunostaining for type II collagen was stronger and more extensive around the chondrocyte-like cells at 5 weeks in the HA + MSC group compared with the other three treatment groups (Figure 4A). Similarly, western blot analysis showed higher levels of type II collagen in the HA + MSC group, relative to the PBS group (Figure 4B). Weak bands of type I collagen were observed in every group, and there were no significant differences between the groups. The bands of matrix metalloproteinase-13 were weaker in the HA + MSC group, relative to the PBS and PBS + MSC groups (Figure $4 \mathrm{~B}$ ). Quantitative analysis confirmed these findings (Figure 4C).

\section{Fluorescent microscopic findings}

A few CFDA-SE-labeled cells were found within the cartilage at 1 week post transplantation of the PBS + MSC group (Figure 5A). The labeled cells gradually disappeared from the cartilage at 3 and 5 weeks post transplantation (Figure 5B, C). In contrast, the labeled cells appeared both adhered to the surface and scattered within the superficial and transitional layers of the cartilage at 1 week post transplantation in the $\mathrm{HA}+\mathrm{MSC}$ group (Figure $5 \mathrm{D}$ ), but they were more frequent in the transitional layer and showed a columnar arrangement at 3 and 5 weeks (Figure 5E, F). The number of labeled cells was higher in the HA + MSC group than in the PBS + MSC group at all the time periods (Figure 5G). These findings suggest that the CFDA-SE-labeled MSCs in HA migrated into the OA cartilage and were associated with the improved metachromasia and histology OA score.

Double immunofluorescence stained sections were evaluated for the distribution of residual chondrocytes, labeled MSCs, and type II collagen in the OA cartilage at 5 weeks post transplantation of HA + MSC injection. Immunostaining for type II collagen was observed in the matrix around residual chondrocytes in the radial layer at 5 weeks (Figure 6B, C), in addition to pericellularly and in the matrix between labeled MSCs in the transitional layer (Figure 6B, D). These findings indicated chondrogenic differentiation of the injected MSCs. 
A

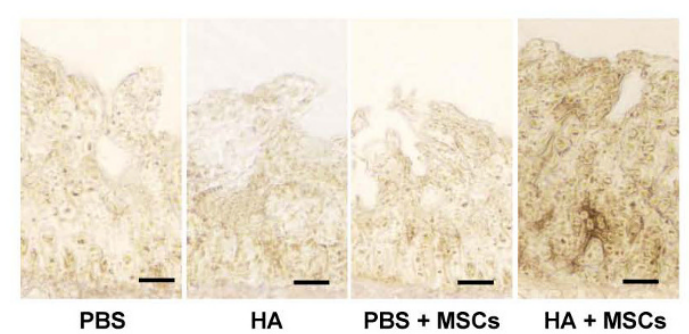

B

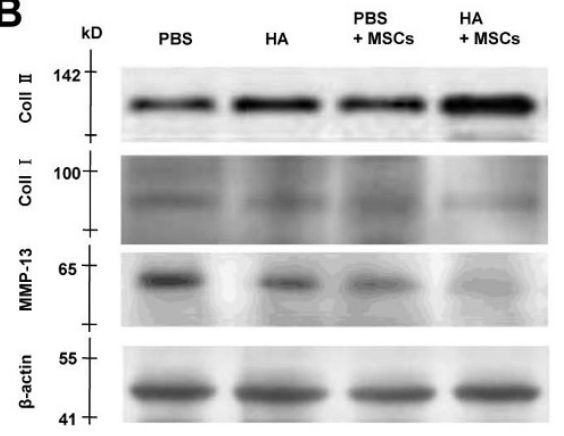

C

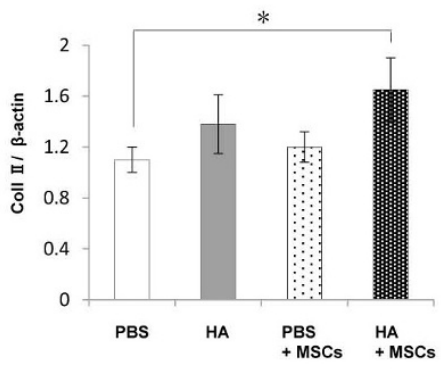

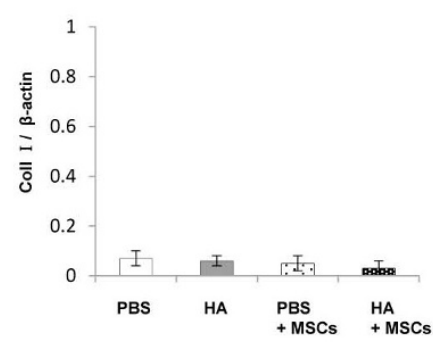

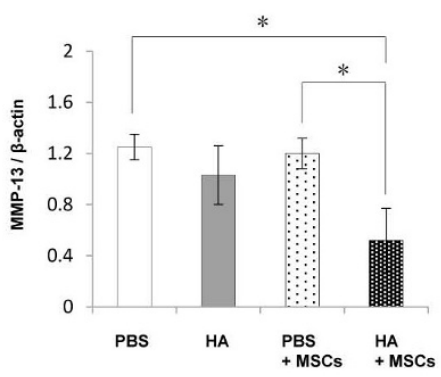

Figure 4 Immunohistochemical and immunoblot analyses. Immunohistochemistry, western blotting, and quantification of type II collagen, type I collagen, and matrix metalloproteinase (MMP)-13 expressions at week 5 after injection. (A) Immunohistochemical analysis for type II collagen. Bar $=50 \mu \mathrm{m}$. (B) Western blot analysis of type II collagen, type I collagen, and MMP-13 (pooled samples in each group, $n=5$ ). (C) Quantitative analysis of the expressions of type II collagen, type I collagen, and MMP-13 relative to the intensity of $\beta$-actin. Data are mean \pm standard deviation of five guinea pigs in each group. ${ }^{*} P<0.05$. HA, hyaluronic acid; MSC, mesenchymal stem cell.

\section{Discussion}

MSCs can be isolated from a variety of mature tissues, and readily expand in culture without losing their multilineage differentiation potential $[4,18,19]$. Moreover, MSCs secrete a broad spectrum of bioactive molecules, collectively known as trophic factors [20]. Previous reports, however, have demonstrated that MSCs represent only 0.01 to $0.001 \%$ of the total nucleated cells within isolated bone marrow aspirates [4] and that the true identity of MSCs is often confused by various isolation and in vitro culture methods [21]. In this regard, preparation of large quantities of autologous MSCs for therapeutic use from small experimental animals is sometimes technologically difficult. Several groups reported recently that MSCs produce molecules that are directly involved in the regulation of the immune
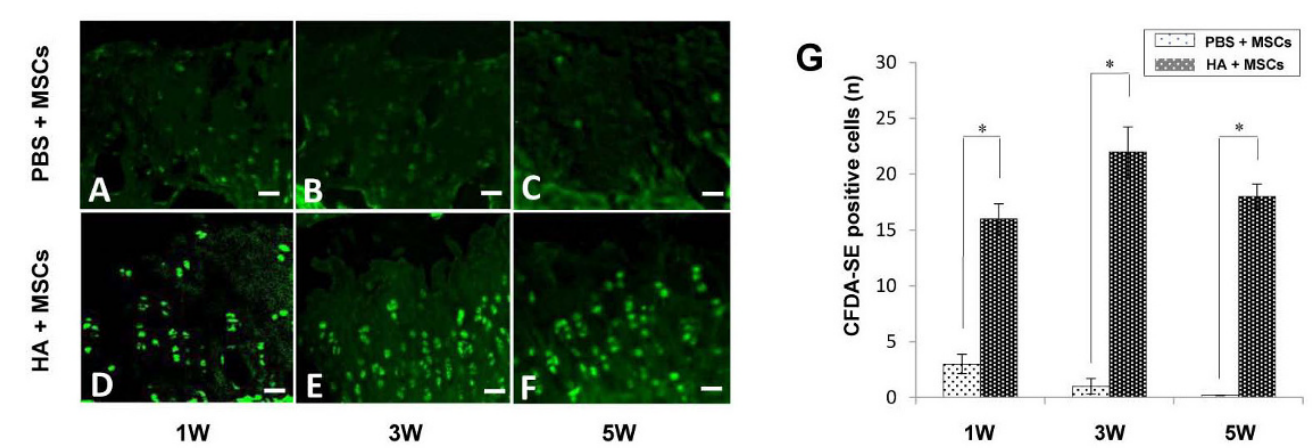

Figure 5 Fluorescent microscopic findings. Fluorescent microscopy for in vivo tracing of injected mesenchymal stem cells (MSCs) at 1, 3, and 5 weeks. (A to C) PBS + MSC group. (D to F) Hyaluronic acid (HA) + MSC group. Bar $=50 \mu \mathrm{m}$. (G) Quantification of the carboxyfluorescein diacetate succinimidyl ester (CFDA-SE)-labeled MSCs. Data are mean \pm standard deviation of five guinea pigs in each group. ${ }^{*} P<0.05$. 

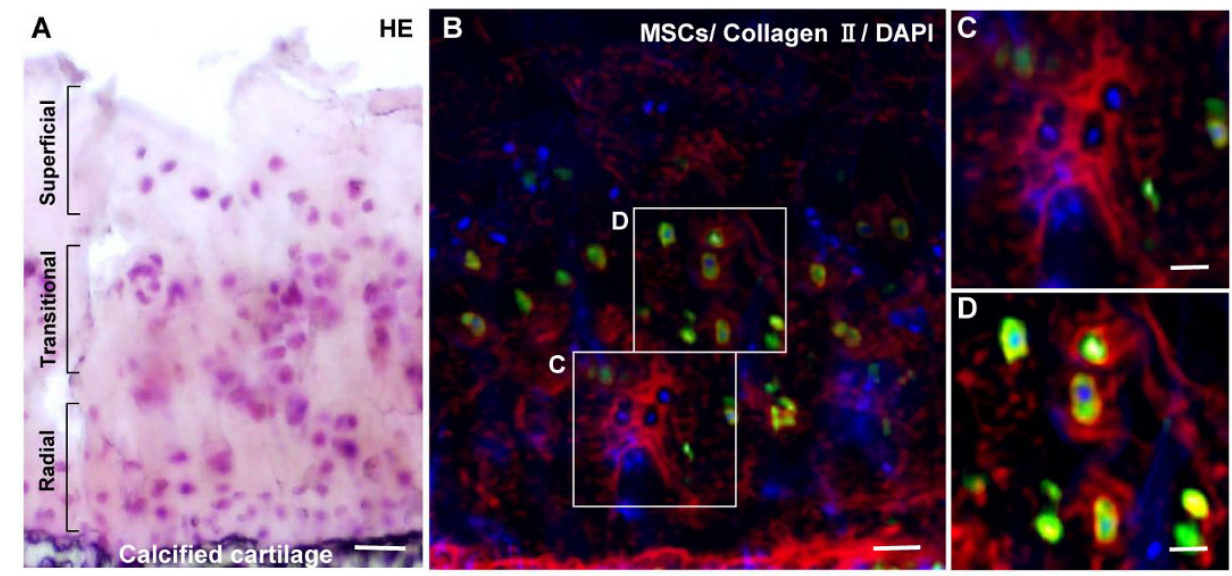

Figure 6 Double immunofluorescence microscopic findings. Photomicrographs showing serial sections of articular cartilage 5 weeks after injection in the hyaluronic acid (HA) + mesenchymal stem cell (MSC) group stained with (A) H \& E and (B to D) double immunofluorescence (red, type II collagen; green, MSCs; blue, 4',6-diamidino-2-phenylindole (DAPI)). (B) corresponds to (A). (C) and (D) are magnified views of the boxes marked in (B). Bar = $100 \mu \mathrm{m}(\mathrm{A}, \mathrm{B}), 50 \mu \mathrm{m}(\mathrm{C}, \mathrm{D})$.

response and have immunosuppressive properties, which may be particularly important in allogeneic MSC transplantation [22,23]. In addition, human MSCs evade xenogeneic immune reactions and inhibit the inflammatory response in collagen-induced arthritis in mice [24]. For these reasons, we used commercially available human MSCs for xenogeneic MSC transplantation in this study. In the present study, the guinea pigs tolerated the cell injections well, and there was no evidence of local inflammation. Frontal histological section of the whole knee joint showed no evidence of synovial hyperplasia. Fluorescence microscopy also identified the presence of the labeled MSCs in the fibrillated cartilage at the medial tibial plateau. A few labeled cells were also detected in the synovial lining and medial meniscus.

In the present study, we evaluated the feasibility of intra-articular injection of MSCs suspended in HA for the treatment of knee OA. Our results at 5 weeks post transplantation showed histologically confirmed partial repair of the articular cartilage, compared with the three other treatment groups. Immunoblot analysis also showed increasing content of type II collagen and low levels of matrix metalloproteinase-13 in the repaired cartilage. In vivo tracing techniques using CFDA-SE labeling and fluorescence microscopy demonstrated that the intra-articularly injected MSCs in HA migrated throughout the osteoarthritic cartilage. Double immunofluorescence analysis also demonstrated strong, although partial, immunostaining of type II collagen around both residual chondrocytes and the injected MSCs after 5 weeks. These results imply intra-articular differentiation of the injected MSCs into chondrocytes and their subsequent proliferation. While $\mathrm{HA}$ is known to influence chondrocyte metabolism, the MSCs may produce trophic factors that could also stimulate the residual chondrocytes.

In our animal study, MSCs possibly produced trophic factors for residual chondrocytes, in addition to differentiating into chondrocytes in the presence of HA. While the biochemical conditions necessary for stimulation of chondrogenesis of MSCs in vitro have been defined [25], little is known about the differentiation of MSCs into chondrocytes and cartilage regeneration in vivo. Several studies investigated chondrogenesis of grafted MSCs within scaffolds in full-thickness cartilage defects in animal models [26,27]. The analyses in these studies showed improved tissue filling and increased matrix staining for type II collagen and proteoglycans 5 to 8 weeks after grafting. The studies' results showed that the transplanted cells secreted type II collagen and contributed to articular cartilage repair. Although simple injection of MSCs was not successful in the repair of cartilage in the present study, MSCs suspended in HA appeared to migrate and proliferate into the transitional layer - and differentiation of these cells into chondrocytes was observed at week 5 after injection. For this treatment group, there was partial repair of cartilage degeneration, both macroscopically and microscopically. The results thus suggest that HA is an important factor in MSC transplantation into the articular cartilage.

There are several advantages for the use of the MSC and HA mixture for intra-articular injection. HA exhibits biological affinity for MSC binding via the transmembrane receptor CD44, which facilitates cell migration through interaction with extracellular HA [28]. HA also increases the chondrogenic activity of 
MSCs [29]. Another study demonstrated that HA coats the surface of the articular cartilage and also locates among the collagen fibrils and sulfated proteoglycans within the cartilage [30]. Furthermore, in vitro experiments indicated that $\mathrm{HA}$ alone enhanced the rate of synovial cell migration, and that HA increased chondrocyte migration in the presence of basic fibroblast growth factor [31]. Considered together, our findings and the results of the above studies emphasize the importance of HA in facilitating the migration, adherence, and differentiation of MSCs onto the OA cartilage.

Previous studies used the MSC-HA combination for cartilage repair in surgically induced OA $[9,10]$, but not for spontaneous OA as was carried out in the present study using the Hartley strain guinea pigs. We compared the difference between disease progression of Hartley guinea pigs knees, with and without transection of the anterior cruciate ligament [32]. Their results showed accelerated cartilage degeneration and higher levels of matrix metalloproteinase- 13 and IL-1 $\beta$, which is a key mediator for OA progression, in the anterior cruciate ligament transection model. These results indicated that the progression of OA may differ between surgically induced and spontaneous OA. In this regard, this animal model of spontaneous OA is considered more suitable for the evaluation of disease-modifying OA treatments.

Our study has certain limitations that should be considered when interpreting the results of the experiments. These include the use of nonphysiologic conditions for xenogeneic MSC transplantation, possible deterioration of the repaired cartilage after injection, a lack of ultrastructural analysis such as morphological evaluation by electron microscopy to clarify the in vivo chondrogenesis of MSCs in OA cartilage after the injection, a lack of re-examination of optimum timing of injection during OA progression, lack of a MSC population isolated from various types of adult mesenchymal tissues and various issues related to the transplantation of autologous or xenogeneic derived stem cells, and the use of HA only instead of including another compound with molecular weight similar to HA. Further studies are needed that include the above points. Should these issues be resolved satisfactorily, then intra-articular injection of MSCs suspended in HA could be a potentially useful therapeutic intervention for the treatment of spontaneous knee OA.

\section{Conclusion}

The results of the present study suggest that intraarticular injection of MSCs with HA is a potentially beneficial therapy for OA. HA exerts cell-binding and cytotactic effects, thus enhancing the migration and proliferation of MSCs. Although further examination of the long-term effects is necessary, this scaffold-free, minimally invasive treatment seems to retard the progression of spontaneous $\mathrm{OA}$ and stimulates the regeneration of articular cartilage.

\section{Abbreviations}

CFDA-SE: carboxyfluorescein diacetate succinimidyl ester; HA: hyaluronic acid; $H$ \& E: hematoxylin and eosin; IL: interleukin; MSC: mesenchymal stem cell; OA: osteoarthritis; PBS: phosphate-buffered saline.

\section{Acknowledgements}

The present work was supported in part by a grant-in-aid for general scientific research to TM from the Ministry of Education, Science and Culture of Japan (grant number 23791632).

\section{Author details}

${ }^{1}$ Department of Orthopaedics and Rehabilitation Medicine, Faculty of Medical Sciences, University of Fukui, Matsuoka-Shimoaizuki 23, Eiheiji, Fukui 910-1193, Japan. ${ }^{2}$ Institute for Science \& Technology in Medicine, Keele University at the RJAH Orthopaedic Hospital, Oswestry SY10 7AG, UK.

\section{Authors' contributions}

MS carried out most of the experiments, interpreted the data, and drafted the manuscript. KU contributed to study design and conception, analysis of data, and drafting of the manuscript. HN and TM contributed to analysis and interpretation of data and drafting of the manuscript. ARG and SW contributed to acquisition of data and drafting of the manuscript. SR contributed to acquisition of data and critical revision of the manuscript. HB contributed to study conception and critical revision of the manuscript. All authors read and approved the final manuscript.

\section{Competing interests}

The authors declare that they have no competing interests.

Received: 15 August 2011 Revised: 29 December 2011

Accepted: 7 February 2012 Published: 7 February 2012

\section{References}

1. Brooks PM: Impact of osteoarthritis on individuals and society: how much disability? Social consequences and health economic implications. Curr Opin Rheumatol 2002, 14:573-577.

2. Friedenstein AJ: Precursor cells of mechanocytes. Int Rev Cytol 1976, 47:327-359.

3. Owen M, Friedenstein AJ: Stromal stem cells: marrow-derived osteogenic precursors. In Cell and Molecular Biology of Vertebrate Hard Tissues. Edited by: Evered D, Hartnett S. Chichester: Wiley; 1998:42-60.

4. Pittenger MF, Mackay AM, Beck SC, Jaiswal RK, Douglas R, Mosca JD, Moorman MA, Simonetti DW, Craig S, Marshak DR: Multilineage potential of adult human mesenchymal stem cells. Science 1999, 284:143-147.

5. Jaiswal N, Haynesworth SE, Caplan Al, Bruder SP: Osteogenic differentiation of purified, culture-expanded human mesenchymal stem cells in vitro. J Cell Biochem 1997, 64:295-312.

6. Mackay AM, Beck SC, Murphy JM, Barry FP, Chichester CO, Pittenger MF: Chondrogenic differentiation of cultured human mesenchymal stem cells from marrow. Tissue Eng 1998, 4:415-428.

7. Wakitani S, Goto T, Pineda SJ, Young RG, Mansour JM, Caplan Al, Goldberg VM: Mesenchymal cell-based repair of large, full-thickness defects of articular cartilage. J Bone Joint Surg Am 1994, 76:579-592.

8. Wakitani S, Imoto K, Yamamoto T, Saito M, Murata N, Yoneda M: Human autologous culture expanded bone marrow mesenchymal cell transplantation for repair of cartilage defects in osteoarthritic knees. Osteoarthritis Cartilage 2002, 10:199-206.

9. Murphy JM, Fink DJ, Hnziker EB, Barry FP: Stem cell therapy in a caprine model of osteoarthritis. Arthritis Rheum 2003, 56:3464-3474.

10. Lee $K B$, Hui JP, Song IC, Ardany L, Lee EH: Injectable mesenchymal stem cell therapy for large cartilage defects - a porcine model. Stem Cells 2007, 25:2964-2971.

11. Bendele AM: Animal models of osteoarthritis. J Musculoskel Neuron Interact 2001, 1:363-376. 
12. Bendele AM, White SL, Hulman JF: Osteoarthritis in guinea pigs: histopathologic and scanning electron microscopic features. Lab Anim Sci 1989, 39:115-121.

13. Sato M, Wada M, Miyoshi N, Imamura Y, Noriki S, Uchida K, Kobayashi S, Yayama T, Negoro K, Fujimoto M, Fukuda M, Baba H: Hydroxyapatite maturity in the calcified cartilage and underlying subchondral bone of guinea pigs with spontaneous osteoarthritis: analysis by Fourier transforms infrared microspectroscopy. Acta Histochem Cytochem 2004, 37:101-107.

14. Urbani S, Caporale R, Lombardini L, Bosi A, Saccardi R: Use of CFDS-SE for evaluating the in vitro proliferation pattern of human mesenchymal stem cells. Cytotherapy 2006, 8:243-253.

15. Hayashi M, Muneta T, Ju YJ, Mochizuki T, Sekiya I: Weekly intra-articular injection of bone morphogenetic protein-7 inhibits osteoarthritis progression. Arthritis Res Ther 2008, 10:R118

16. Kawamoto T, Shimizu M: A method for preparing 2- to 50-micron-thick fresh-frozen sections of large samples and undecalcified hard tissues. Histhochem Cell Biol 2000, 113:331-339.

17. Armstrong $L$, Read R, Ghosh P: The effects of intraarticular hyaluronan on cartilage and subchondral bone changes in an ovine model of early osteoarthritis. J Rheumatol 1994, 21:680-688.

18. Kolf CM, Cho E, Tuan RS: Mesenchymal stromal cells. Biology of adult mesenchymal stem cells: regulation of niche, self-renewal and differentiation. Arthritis Res Ther 2007, 9:204

19. Chen FH, Rousche KT, Tuan RS: Technology insight: adult stem cells in cartilage regeneration and tissue engineering. Nat Clin Pract Rheumatol 2006, 3:373-382.

20. Caplan Al, Dennis JE: Mesenchymal stem cells as trophic mediators. J Cell Biochem 2006, 98:1076-1084.

21. Salem HK, Thiemermann C: Mesenchymal stromal cells: current understanding and clinical status. Stem Cells 2010, 28:585-596.

22. EL-Badri NS, Maheshwari A, Sanberg PR: Mesenchymal stem cells in autoimmune disease. Stem Cells Dev 2004, 13:463-472.

23. Prittenger MF, Martin BJ: Mesenchymal stem cells and their potential as cardiac therapeutics. Circ Res 2004, 95:9-20.

24. Gonzalez MA, Rey EG, Rico L, Buscher D, Delgado M: Treatment of experimental arthritis by inducing immune tolerance with human adipose-derived mesenchymal stem cells. Arthritis Rheum 2009, 60:1006-1019.

25. Johnstone B, Hering TM, Caplan Al, Goldberg VM, Yoo JU: In vitro chondrogenesis of bone marrow-derived mesenchymal progenitor cells. Exp Cell Res 1998, 238:265-272.

26. Wilke MM, Nydam DV, Nixon AJ: Enhance early chondrogenesis in articular defects following arthroscopic mesenchymal stem cell implantation in an equine model. J Orthop Res 2006, 25:913-925.

27. Chang F, Ishii T, Yanai T, Mishima H, Akaogi H, Ogawa T, Ochiai N: Repair of large full-thickness articular cartilage defects by transplantation of autologous uncultured bone-marrow-derived mononuclear cells. J Orthop Res 2007, 26:18-26.

28. Hui Z, Mitsuhashi N, Klein A, Barsky LW, Weinberg K, Barr ML, Demetriou A, Wu GD: The role of the hyaluronan receptor CD44 in mesenchymal stem cell migration in the extracellular matrix. Stem cells 2005, 24:928-935.

29. Kavalkovich KW, Boynton RE, Murphy JM, Barry F: Chondrogenic differentiation of human mesenchymal stem cells within an alginate layer culture system in vitro. Cell Develop Biol Animal 2002, 38:457-466.

30. Maniwa S, Ochi M, Motomura T, Nishikori T, Chen J, Naora H: Effects of hyaluronic acid and basic fibroblast growth factor on motility of chondrocytes and synovial cells in culture. Acta Orthop Scand 2001, 72:299-303.

31. Yoshioka M, Shimizu C, Harwood FL, Coutts RD, Amiel D: The effects of hyaluronan during the development of osteoarthritis. Osteoarthritis Cartilage 1997, 5:251-260.

32. Wei L, Fleming BC, Sun X, Teeple E, Wu W, Jay GD, Elsaid KA, Luo J, Machan JT, Chen Q: Comparison of different biomarkers of osteoarthritis with and without posttraumatic injury in the Hartley guinea pig model. J Orthop Res 2010, 28:900-906.

doi:10.1186/ar3735

Cite this article as: Sato et al: Direct transplantation of mesenchymal stem cells into the knee joints of Hartley strain guinea pigs with spontaneous osteoarthritis. Arthritis Research \& Therapy 2012 14:R31.

\section{Submit your next manuscript to BioMed Central and take full advantage of:}

- Convenient online submission

- Thorough peer review

- No space constraints or color figure charges

- Immediate publication on acceptance

- Inclusion in PubMed, CAS, Scopus and Google Scholar

- Research which is freely available for redistribution

Submit your manuscript at www.biomedcentral.com/submit 